\title{
ERRATUM TO: "EPR SPECTROSCOPY OF TETRABORATE GLASSES, DOPED WITH Mn AND Cu”, CTBo 2010, 33 (suppl. A) 171-175
}

\author{
BOHDAN V. PADLYAK ${ }^{1,2}$, ADAM DRZEWIECKI ${ }^{1}$, OLEKSANDR O. SMYRNOV ${ }^{1}$ \\ ${ }^{1}$ Division of Spectroscopy of Functional Materials, Institute of Physics, University of Zielona Góra, 4a Szafrana Str., 65-516 Zielona Góra, Poland \\ ${ }^{2}$ Section of Spectroscopy, Institute of Physical Optics, 23 Dragomanov Str., 79-005 Lviv, Ukraine
}

Received October 12, 2012; accepted October 17, 2012; published online December 31, 2012

After publication of this paper, during the sequence of further optical measurements of the same samples we noticed that one of the samples was incorrectly labeled. In order to correct this mistake, published results were

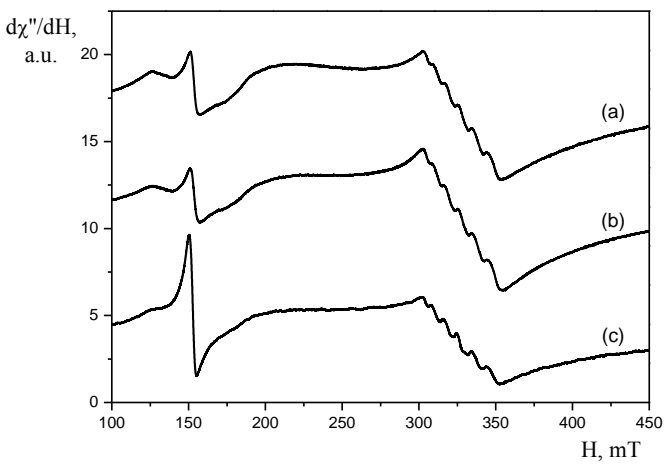

checked and corrected. Figure 1 with corresponding caption, caption for Figure 2 and Tables 1-3 changes as follows:

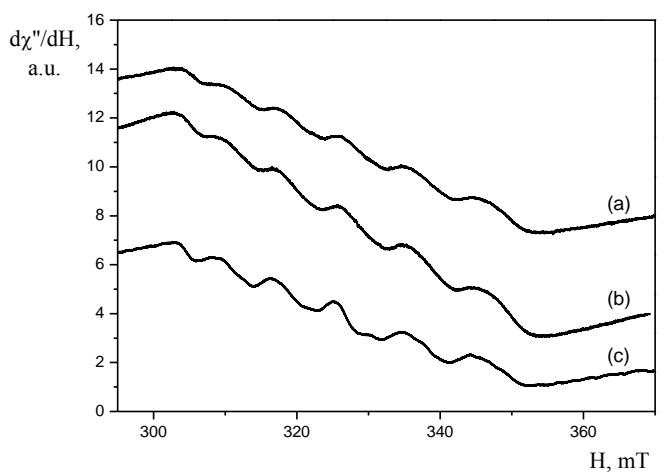

Fig. 1. The room temperature EPR spectra of the KLTB:Mn (a) and LTB:Mn (b, c) glasses, containing 0.4 mol.\% (c) and 1.6 mol.\% (a, b) of the $\mathrm{MnO}_{2}$ compound. Left side - complete EPR spectra, right side - central part of the EPR spectra.

Fig. 2. The room temperature EPR spectra of the KLTB:Cu (a, b) and LTB:Cu (c, d) glasses, containing 0.4 mol.\% (b, d) and 1.6 mol.\% (a, c) of the $\mathrm{CuO}$ compound. Left side - complete EPR spectra, right side - central part of the EPR spectra.

Table 1. The parameters of EPR signals of the $\mathrm{Fe}^{3+}$ centres in tetraborate glasses

\begin{tabular}{cccc}
\hline Glass composition & Impurity amount (mol.\%) & $g$-factor value & EPR linewidth, $\Delta H_{\mathrm{pp}}(\mathrm{mT})$ \\
\hline $\mathrm{Li}_{2} \mathrm{~B}_{4} \mathrm{O}_{7}: \mathrm{Cu}$ & 0.4 & $4.31 \pm 0.01$ & $4.0 \pm 0.5$ \\
& 1.6 & $4.27 \pm 0.01$ & $3.9 \pm 0.5$ \\
$\mathrm{KLiB}_{4} \mathrm{O}_{7}: \mathrm{Cu}$ & 0.4 & $4.29 \pm 0.01$ & $3.6 \pm 0.5$ \\
& 1.6 & $4.28 \pm 0.01$ & $3.7 \pm 0.5$ \\
$\mathrm{Li}_{2} \mathrm{~B}_{4} \mathrm{O}_{7}: \mathrm{Mn}$ & 0.4 & $4.31 \pm 0.01$ & $5.4 \pm 0.5$ \\
$\mathrm{KLiB}_{4} \mathrm{O}_{7}: \mathrm{Mn}$ & 1.6 & $4.26 \pm 0.01$ & $6.2 \pm 0.5$ \\
\end{tabular}


Table 2. The parameters of EPR spectra of the $\mathrm{Mn}^{2+}$ centres in tetraborate glasses

\begin{tabular}{|c|c|c|c|c|c|c|c|}
\hline Glass composition & \multicolumn{2}{|c|}{$\mathrm{MnO}_{2}$ amount (mol.\%) } & \multicolumn{2}{|l|}{$g_{\text {iso }}$} & $A_{\text {iso }}(\mathrm{mT})$ & \multicolumn{2}{|c|}{$\Delta B_{\text {iso }}(\mathrm{mT})$} \\
\hline & \multicolumn{2}{|c|}{0.4} & \multicolumn{2}{|l|}{$2.03 \pm 0.01$} & $8.9 \pm 0.5$ & \multicolumn{2}{|c|}{$6.2 \pm 0.5$} \\
\hline $\mathrm{Li}_{2} \mathrm{~B}_{4} \mathrm{O}_{7}: \mathrm{Mn}$ & \multicolumn{2}{|c|}{1.6} & \multicolumn{2}{|l|}{$2.03 \pm 0.01$} & $8.9 \pm 0.5$ & \multicolumn{2}{|c|}{$6.9 \pm 0.5$} \\
\hline $\mathrm{KLiB}_{4} \mathrm{O}_{7}: \mathrm{Mn}$ & \multicolumn{2}{|c|}{1.6} & \multicolumn{2}{|l|}{$2.03 \pm 0.01$} & $8.7 \pm 0.5$ & \multicolumn{2}{|c|}{$7.0 \pm 0.5$} \\
\hline Glass composition & $\begin{array}{c}\mathrm{CuO} \\
\text { amount } \\
(\mathrm{mol} \%)\end{array}$ & $\overline{g_{\|}}$ & $g_{\perp}$ & $\begin{array}{c}A_{\|} \\
(\mathrm{mT})\end{array}$ & $\begin{array}{c}A_{\perp} \\
(\mathrm{mT})\end{array}$ & $\begin{array}{l}\Delta B_{p p}^{\|} \\
(\mathrm{mT})\end{array}$ & $\begin{array}{l}\Delta B_{p p}^{\perp} \\
(\mathrm{mT})\end{array}$ \\
\hline \multirow{2}{*}{$\mathrm{Li}_{2} \mathrm{~B}_{4} \mathrm{O}_{7}: \mathrm{Cu}$} & 0.4 & $2.31 \pm 0.01$ & $2.06 \pm 0.01$ & $15.5 \pm 0.5$ & $2.9 \pm 0.5$ & $5.1 \pm 0.5$ & $1.7 \pm 0.5$ \\
\hline & 1.6 & $2.31 \pm 0.01$ & $2.06 \pm 0.01$ & $14.9 \pm 0.5$ & $2.7 \pm 0.5$ & $5.7 \pm 0.5$ & $1.7 \pm 0.5$ \\
\hline \multirow{2}{*}{$\mathrm{KLiB}_{4} \mathrm{O}_{7}: \mathrm{Cu}$} & 0.4 & $2.31 \pm 0.01$ & $2.06 \pm 0.01$ & $15.2 \pm 0.5$ & $2.9 \pm 0.5$ & $5.9 \pm 0.5$ & $1.7 \pm 0.5$ \\
\hline & 1.6 & $2.31 \pm 0.01$ & $2.06 \pm 0.01$ & $15.2 \pm 0.5$ & $2.8 \pm 0.5$ & $5.9 \pm 0.5$ & $1.9 \pm 0.5$ \\
\hline
\end{tabular}

